\title{
Some Rigorous Results on the Sherrington-Kirkpatrick Spin Glass Model
}

\author{
M. Aizenman $\star$ J. L. Lebowitz ${ }^{\star \star}$, and D. Ruelle ${ }^{\star \star}$ \\ Departments of Mathematics and Physics, Rutgers University, New Brunswick, NJ 08903, USA \\ Dedicated to Walter Thirring on his $60^{\text {th }}$ birthday
}

\begin{abstract}
We prove that in the high temperature regime $(T / J>1)$ the deviation of the total free energy of the Sherrington-Kirkpatrick (S-K) spin glass model from the easily computed $\log \operatorname{Av}\left(Z_{N}(\{\beta J\})\right)$ converges in distribution, as $N \rightarrow \infty$, to a (shifted) Gaussian variable. Some weak results about the low temperature regime are also obtained.
\end{abstract}

\section{Introduction}

We consider the Sherrington-Kirkpatrick [1] spin glass model, with the mean field Hamiltonian

$$
H=-\sum_{1 \leqq i<j \leqq N} \frac{J_{i j}}{\sqrt{N}} \sigma_{i} \sigma_{j},
$$

where the spins $\sigma_{1}, \ldots, \sigma_{N}$ take values \pm 1 , and the $J_{i j}$ 's are independent identically distributed random variables with mean zero and variance $J^{2}$. (The randomness is reflected in the unusual scaling factor $1 / \sqrt{N}$ in $H$.) It is believed that this model has trivial behavior at high temperatures (for $\beta J<1$ ) while at low temperatures $(\beta J>1)$ it has a rich structure of "Gibbs states" or "valleys" described by Parisi's Replica Symmetry Breaking solution; see [2] for a review of the history, the current status, and an extended list of references - on this and related subjects.

While the Parisi solution is widely believed to be exact, it is not claimed to be rigorous. In fact, we have not found in the literature a complete treatment of even the high temperature region. The main purpose of this note is to provide a full description of the free energy in that regime. We supplement the existing analysis by deriving the limiting probability law of the fluctuations in the total free energy. In addition, the results of Sherrington and Kirkpatrick [1] and Thouless et al. [3]

\footnotetext{
* Research supported in part by the NSF grant PHY-8605164. Present address: Courant Institute of Mathematical Sciences, 251 Mercer St., New York, NY 10012, USA

$\star \star$ Research supported in part by the NSF grant DMR 86-12369

$\star \star \star$ On leave from Institut des Hautes Etudes Scientifiques, Bures-Sur-Yvette, France
} 
on the average value of the free energy per site are given here a rigorous proof. Also presented are some weak but explicit bounds pertaining to the low temperature region, on an order parameter which is similar to that of Edwards and Anderson, and on the energy per site.

\section{Assumptions and Statement of Results}

We make the standing assumption that the distribution of the $J_{i j}$ is symmetric with respect to zero, and has moments of all orders. In particular we write

$$
\left\langle\left\langle J_{i j}^{2}\right\rangle=J^{2},\right.
$$

denoting by $\left\langle\langle\ldots\rangle\right.$, or alternatively by $\operatorname{Av}(\ldots)$, the average over the $J_{i j}$ 's. This is to be distinguished from the averages over the $\sigma_{i}$ 's - of which the a-priori (equal weight) average is denoted here by tr, and the thermal average (corresponding to fixed $\left.\left\{\beta J_{i j}\right\}\right)$ is denoted by $\langle\ldots\rangle$. Examples to keep in mind are that of $J_{i j}$ 's independent Gaussian variables, with

$$
P\left(d J_{i j}\right)=\frac{1}{\sqrt{2 \pi} J}\left(\exp -J_{i j}^{2} / 2 J^{2}\right) d J_{i j}
$$

and the discrete case, $J_{i j}= \pm 1$, with

$$
P\left(d J_{i j}\right)=\frac{1}{2}\left(\delta\left(J_{i j}-J\right)+\delta\left(J_{i j}+J\right)\right) d J_{i j} .
$$

Of principal interest is the distribution of the partition function

$$
Z_{N}(\{\beta J\})=\sum_{\sigma_{1}} \ldots \sum_{\sigma_{N}} \exp -\beta H=2^{N} \operatorname{tr} \exp \sum_{i<j} \frac{\beta J_{i j}}{\sqrt{N}} \sigma_{i} \sigma_{j},
$$

where $\{\beta J\}$ denotes the family of couplings $\beta J_{i j}$.

Note that by the symmetry of the distribution of the $J_{i j}$ 's we have

$$
\left\langle\left\langle Z_{N}\right\rangle\right\rangle=2^{N} \prod_{i<j}\left\langle\left\langle\cosh \frac{\beta J_{i j}}{\sqrt{N}}\right\rangle\right\rangle,
$$

so that

$$
\log \langle\langle Z\rangle\rangle=N\left[\log 2+\frac{1}{4} \beta^{2} J^{2}\right]-\frac{1}{4} \beta^{2} J^{2}+\frac{\beta^{4} \lambda}{2 \cdot 4 !}+O\left(\frac{1}{N}\right),
$$

with $\lambda=\left\langle\left\langle J_{12}^{4}\right\rangle\right\rangle-3\left\langle\left\langle J_{12}^{2}\right\rangle\right\rangle^{2}$ (which vanishes in the Gaussian case). Here, and in what follows, the subscript $N$ is sometimes omitted.

The calculation of the mean free energy, which is proportional to $P_{N}(\beta)$ $=1 / N\langle\langle\log Z\rangle\rangle$ is, of course, more difficult. In addition, the question of the sharpness of the free energy per site, in the sense of its convergence in distribution to a constant as $N \rightarrow \infty$ [as in (2.11)], is nontrivial for the SK model. In this respect, a special situation exists whenever

$$
\lim _{N \rightarrow \infty} \frac{1}{N}\langle\langle\log Z\rangle\rangle=\lim _{N \rightarrow \infty} \frac{1}{N} \log \langle\langle Z\rangle\rangle
$$


(i.e. the "quenched" and the "annealed" free energies agree) - which is predicted by Sherrington and Kirkpatrick [1] and Thouless et al. [3] to hold for $\beta J<1$. In such case, the sharpness of the distribution of the free energy density may be deduced from just the mean value. [However, it is generally expected that the distribution remains "sharp", i.e. the free energy density has the "self averaging" property, even when (2.6) fails.] of

It turns out that (2.6) is equivalent to the vanishing, as $N \rightarrow \infty$, of the mean value

$$
\tau_{N}(\{\beta J\})=\frac{2}{N(N-1)} \sum_{1 \leqq i<j \leqq N}\left\langle\sigma_{i} \sigma_{j}\right\rangle^{2}
$$

(see Proposition 4.1). The limit of the corresponding mean, if it exists, defines an order parameter which is somewhat similar to that of Edwards and Anderson.

The following statement summarizes the high-temperature behavior of the free energy to leading order in $N$.

Proposition 2.1. For all $\beta J<1$,

$$
\lim _{N \rightarrow \infty}\left\langle\left\langle\tau_{N}(\{\beta J\})\right\rangle\right\rangle=0
$$

ii) the mean free energy satisfies

$$
\lim _{N \rightarrow \infty} \frac{1}{N}\langle\langle\log Z\rangle\rangle=\log 2+\frac{1}{4} \beta^{2} J^{2}=\lim _{N \rightarrow \infty} \frac{1}{N} \log \langle\langle Z\rangle\rangle
$$

and iii) the free energy per site converges to its mean value, in probability,

$$
\operatorname{Prob}_{J}\left(\left|\frac{1}{N} \log Z-\left(\log 2+\frac{1}{4} \beta^{2} J^{2}\right)\right| \geqq \varepsilon\right) \underset{N \rightarrow \infty}{\longrightarrow} 0 \text { for all } \varepsilon>0,
$$

and also in the $L^{p}$ sense for each $p<\infty$.

We shall go beyond (2.10) by showing that the fluctuations of the total free energy, i.e. $\log (Z /\langle Z\rangle\rangle)$, are of order one, and converge in distribution to a (shifted) Gaussian. (Actually, we need this analysis for the proof of Proposition 2.1.)

To describe the fluctuations let us decompose the free energy as follows,

$$
Z_{N}(\{\beta J\})=2^{N}\left[\prod_{i<j} \cosh \frac{\beta J_{i j}}{\sqrt{N}}\right] \cdot \hat{Z}_{N}, \quad \text { with } \quad \hat{Z}_{N}=\operatorname{tr} \prod_{i<j}\left(1+\sigma_{i} \sigma_{j} \tanh \frac{\beta J_{i j}}{\sqrt{N}}\right) .
$$

Proposition 2.2. For $\beta J<1$,

i) $\hat{Z}_{N}$ tends in distribution, when $N \rightarrow \infty$, to the log-normal variable

$$
\widehat{T}=\exp \left(v-\frac{1}{2}\left\langle v^{2}\right\rangle\right),
$$

where $v$ is a Gaussian random variable with variance

and

$$
\left\langle v^{2}\right\rangle=-\frac{1}{2}\left[\log \left(1-\beta^{2} J^{2}\right)+\beta^{2} J^{2}+\frac{1}{2} \beta^{4} J^{4}\right]=\sum_{k=3}^{\infty} \frac{1}{2 k}\left(\beta^{2} J^{2}\right)^{k},
$$

ii) $Z_{N} /\left\langle\left\langle Z_{N}\right\rangle\right\rangle$ tends in distribution, when $N \rightarrow \infty$, to

$$
T=\exp \left(u-\frac{1}{2}\left\langle u^{2}\right\rangle\right)
$$


where $u$ is a Gaussian random variable with variance

$$
\left\langle u^{2}\right\rangle=-\frac{1}{2}\left[\log \left(1-\beta^{2} J^{2}\right)+\beta^{2} J^{2}-\lambda \beta^{4} / 4\right] .
$$

Remarks. The convergence in distribution, of a sequence of random variables $X_{N}$ to a variable $Y\left(\mathscr{D}-\lim X_{N}=Y\right.$, or $\left.X_{N} \stackrel{\mathscr{D}}{\longrightarrow} Y\right)$, means that for all intervals $[a, b]$

$$
\operatorname{Prob}\left(a \leqq X_{N} \leqq b\right) \rightarrow \operatorname{Prob}(a \leqq Y \leqq b),
$$

provided $\operatorname{Prob}(Y=a)=\operatorname{Prob}(Y=b)=0$, i.e. provided the limiting density does not have a $\delta$ function at $Y=a$ or $b$.

A well known criterion is [4]:

C1) If the moments of a sequence of random variables $X_{N}$ converge to the moments of $V$, i.e.

$$
\left\langle X_{N}^{n}\right\rangle \underset{N \rightarrow \infty}{\longrightarrow}\left\langle V^{n}\right\rangle \text { for } n=1,2,3, \ldots
$$

then also

$$
X_{N} \stackrel{\mathscr{D}}{\longrightarrow} V,
$$

provided the distribution of $V$ is uniquely determined by its moments. A sufficient condition for the latter is that $\left\langle V^{n}\right\rangle /\left[\varepsilon^{n} n !\right] \rightarrow 0$, for each $\varepsilon>0$.

In our case, the moments of the limiting distribution $T$ are given by

$$
E\left(T^{n}\right)=\left(\frac{\exp \left(-\frac{1}{2} \beta^{2} J^{2}+\frac{1}{8} \lambda \beta^{4}\right)}{\sqrt{1-\beta^{2} J^{2}}}\right)^{\frac{n(n-1)}{2}} .
$$

Their rate of increase with $n$ is such that criterion C1 does not apply - the lognormal distribution is actually a known example of a law which is not uniquely determined by its moments (see Derrida [5] for specific examples of other distributions with such moments, and a discussion of this point in a relevant context). So even had all the moments of $Z_{N} /\left\langle\left\langle Z_{N}\right\rangle\right\rangle$ converged to (2.16) for $\beta J<1$, that would not be sufficient to prove (2.2). In fact however, as pointed out to us by Derrida, for each $\beta J$ the higher moments of $Z_{N} /\left\langle\left\langle Z_{N}\right\rangle\right\rangle$, i.e. from a certain value $n(\beta)<\infty$, diverge as $N \rightarrow \infty$. The study of the distribution of the free energy has therefore to be based an other techniques. [Nevertheless, it is interesting to note that $\left\langle\left\langle Z_{N}^{2}\right\rangle\right\rangle\left\langle\left\langle Z_{N}\right\rangle\right\rangle^{2} \rightarrow E\left(T^{2}\right)$ for $\beta J<1$.]

As a notion of convergence, $\mathscr{D}$-convergence is one of the weakest. In particular, in itself it does not imply the convergence of the mean values. However, with some extra compactness - like in the following criterion [4], it suffices.

C2) If $X_{N} \stackrel{\mathscr{D}}{\longrightarrow} Y$, while for some $\varepsilon>0\left\langle\left\langle\left|X_{N}\right|^{1+\varepsilon}\right\rangle\right\rangle$ stays bounded uniformly in $N$, then also $\left\langle\left\langle X_{N}\right\rangle\right\rangle \rightarrow\langle\langle Y\rangle$.

This criterion applies to the leading order of the free energy, i.e. the free energy per site (Sect. 4), and enables us to deduce Eq. (2.9) from the results of Proposition 2.2.

Finally, we present some weak low temperature results. To place them in a context, let us first note some implications of well known basic principles. First, in 
any random system, if a function $g(\cdot)$ defined on $[0, \infty)$ satisfies

$$
\limsup _{N \rightarrow \infty} \frac{\beta^{-1}}{N} \log \left\langle\left\langle Z_{N}\right\rangle \leqq g(\beta),\right.
$$

then also the following bound holds

$$
\mathscr{D}-\limsup _{N \rightarrow \infty} \frac{\beta^{-1}}{N} \log Z_{N}(\{\beta J\}) \leqq g^{*}(\beta),
$$

where $g^{*}(\beta)$ is the pointwise largest function which is: i) nonincreasing in $\beta$, and ii) satisfies $g^{*}(\beta) \leqq g(\beta)$, for all $\beta \geqq 0$. An inequality involving " $\mathscr{D}$-lim sup" [as in (2.18)], or " $\mathscr{D}$-lim inf," means here that the probability measure of the set of $J$ 's for which the inequality is violated tends to zero.

The bound (2.18) is derived in two steps. First one may note that such an inequality with $g(\cdot)$, instead of $g^{*}(\cdot)$, is implied by a simple consideration of the mean value of $Z_{N}$ [see (4.9)]. The improvement represented by $g^{*}$ is based on a monotonicity statement which is implied by the nonnegativity of the entropy:

$$
\begin{aligned}
\frac{\partial}{\partial \beta} \beta^{-1} \log Z_{N}(\{\beta J\}) & =-\beta^{-2}\left[\log Z_{N}(\{\beta J\})-\beta\left\langle H_{J}\right\rangle_{\beta J}\right] \\
& =\beta^{-2} \sum_{\sigma} \varrho_{\{\beta J\}}(\sigma) \log \varrho_{\{\beta J\}}(\sigma) \leqq 0,
\end{aligned}
$$

where $\varrho_{\{\beta J\}}(\sigma)=e^{-\beta H(\sigma ; J)} / Z_{N}(\{\beta J\})$ is the Gibbs state corresponding to $\{J\}$, and the inequality holds since $\varrho(\sigma)<1$.

For the systems considered here the simple calculation (2.5) shows that (2.17) is satisfied by $g(\beta)=(\log 2) \beta^{-1}+\beta J^{2} / 4$. The above considerations involving $g^{*}(\cdot)$ suffice to imply that the free energy cannot satisfy (2.9) for $\beta J>2 \sqrt{\log 2} \cong 1.39$ (which is, however, somewhat far from the generally expected critical value $\left.\beta_{c} J=1\right)$.

Another quantity of interest is the ground state energy (per site): $e_{0}(\{J\})$ $=\min _{\sigma} H(\sigma ; J) / N$. Simple thermodynamic considerations related to the above show that

$$
\mathscr{D}-\limsup _{N \rightarrow \infty}\left|e_{0}(\{J\})\right| \leqq \sqrt{\log 2} J \cong 0.83 J
$$

Since the order parameter defined in (2.7) also satisfies the relation

$$
\left\langle\left\langle\tau_{N}(\{\beta J\})\right\rangle \geqq 1-\frac{\left\langle\left\langle 2\left|e_{0}(\{J\})\right| \gg\right\rangle\right.}{\beta J^{2}}+O\left(\frac{1}{\sqrt{N}}\right)\right.
$$

[see (4.14)], one has bounds which show explicitly that it does not vanish at low temperatures [additional information on its behavior near $\beta=1$ is provided by the related inequality (4.12), of Proposition 4.2].

The result presented below provides some bounds which tend to be complementary to the above inequalities.

Proposition 2.3. For all $\beta$,

$$
\underset{D}{\mathscr{D}-\liminf } \frac{1}{N} \log Z_{N} \geqq \log 2+\int_{0}^{1} d u \log \cosh \left(\sqrt{\frac{2 u}{\pi}} \beta J\right) .
$$


The implication of (2.22) for the ground state energy (obtained by letting $\beta \rightarrow \infty$ ) is

$$
\mathscr{D}-\liminf _{N \rightarrow \infty}\left|e_{0}\right| \geqq \sqrt{\frac{2}{\pi}} \frac{2}{3} J \cong 0.53 J .
$$

Making a mild use of Wigner's "semicircle law" one can get a better bound for $e_{0}$ :

Proposition 2.44. For the Gaussian ensemble (2.1),

$$
\mathscr{D}-\liminf _{N \rightarrow \infty}\left|e_{0}\right| \geqq \frac{2}{\pi} J \cong 0.64 J
$$

The restriction to the Gaussian case is expected to be just a matter of convenience for the proof. The value produced by numerical studies is $\lim _{N \rightarrow \infty}\left|e_{0}\right| \cong 0.76 J[6,7]$. While there is obviously room for improvement, let us note that the energy densities of typical configurations accessed by states in the high temperature regime satisfy

$$
-e \approx \frac{1}{2} \beta J^{2} \leqq 0.5 J
$$

so that the bounds in (2.22)-(2.24) do reflect on the the low temperature region.

\section{The Fluctuations in the Total Free Energy at High Temperatures}

In this section we analyze the limiting distribution of the random variable $Z_{N}(\{\beta J\})$. We start by presenting a convergence criterion useful for our purpose [4].

C3) If a given sequence of variables $X_{N}$ can be approximated arbitrarily well in the $L^{2}$ sense by $\mathscr{D}$-convergent sequences, i.e. if for each $\varepsilon>0$ there exist random variables $X_{N}^{(\varepsilon)}$ and $Y^{(\varepsilon)}$ such that

$$
\left\|X_{N}-X_{N}^{(\varepsilon)}\right\|_{2} \leqq \varepsilon
$$

and

ii)

$$
X_{N}^{(\varepsilon)} \stackrel{\mathscr{D}}{\longrightarrow} Y^{(\varepsilon)} \quad(\text { as } N \rightarrow \infty),
$$

then the following limits exist and are equal

$$
\mathscr{D}-\lim _{N \rightarrow \infty} X_{N}=\mathscr{D}-\lim _{\varepsilon \rightarrow 0} Y^{(\varepsilon)} \text {. }
$$

Let us now turn to the quantity $\hat{Z}_{N}$, defined in (2.12). Expanding the product, and performing the tr, one arrives at the following expression

$$
\hat{Z}=\sum_{\partial \Gamma=\emptyset} w(\Gamma)
$$

with

$$
w(\Gamma)=\prod_{b, \text { edges of } \Gamma} \tanh \left(\frac{\beta J_{b}}{\sqrt{N}}\right) .
$$


The summation in (3.1) is over all the graphs with vertices in $\{1,2, \ldots, N\}$ which are simple and closed, i.e. no bond (i.e. edge) is repeated, and the set

$$
\partial \Gamma=\{i \in\{1, \ldots, N\} \mid i \text { belongs to an odd number of edges of } \Gamma\}
$$

is empty.

We shall also encounter here graphs with multiplicities. In general, for a graph $\Gamma$, we denote by $|\Gamma|$ the number of its bonds, counted with their multiplicities in the graph.

The following result indicates the origin of the Gaussian distribution which appears in Proposition 2.2, and demonstrates the way we apply the above convergence criteria.

Lemma 3.1. With $\gamma$ ranging over simple loops on the vertex set $\{1, \ldots, N\}$, let

$$
V_{N}(J)=\sum_{\gamma, \text { simple loops }} w(\gamma)
$$

then, for $\beta J<1$

$$
V_{N} \underset{N \rightarrow \infty}{\stackrel{\mathscr{D}}{\longrightarrow}} v
$$

where $v$ is a Gaussian random variable of variance given by (2.14). Moreover, the sum of $w(\gamma)^{2}$ converges to a constant:

$$
\sum_{\gamma} w(\gamma)^{2} \underset{N \rightarrow \infty}{\longrightarrow}\left\langle v^{2}\right\rangle
$$

where the convergence is both in $\mathscr{D}$ and in $L^{2}$, and

$$
\operatorname{Prob}\left(\max _{\gamma}\{|w(\gamma)|\} \geqq \frac{1}{N^{3 / 2-\varepsilon}}\right) \underset{N \rightarrow \infty}{\longrightarrow} 0
$$

for each $\varepsilon>0$. By implication,

$$
\sum|w(\gamma)|^{k} \stackrel{\mathscr{D}}{\longrightarrow} 0 \text { for each } k>2 .
$$

Proof. The main result here is (3.3) which will be proven first. Our strategy is to split the sum over the graphs into two parts,

$$
\sum_{\gamma} w(\gamma)=\sum_{\gamma:|\gamma| \leqq k} w(\gamma)+\sum_{\gamma:|\gamma|>k} w(\gamma) \equiv F_{\leqq k}+F_{>k},
$$

of which $F_{>k}$ will be shown to be of a small $L^{2}$ norm, and $F_{\leqq k}$ will be shown to converge on the basis of its moments. By Wick's identity for the moments of the Gaussian distribution, and the criteria mentioned above it suffices to show

i)

$$
\left\|F_{>k}\right\|_{2} \leqq \varepsilon_{k},
$$

uniformly in $N$, with $\varepsilon_{k} \rightarrow 0$ (as $\left.k \rightarrow \infty\right)$.

ii)

$$
\lim _{k \rightarrow \infty} \lim _{N \rightarrow \infty}\left\langle\left\langle F_{\leqq k}^{2}\right\rangle\right\rangle=\left\langle v^{2}\right\rangle,
$$

and (the heart of the matter) - 
iii) the quantities

$$
R_{k, n}(N)=\left\langle\left\langle\left(F_{\leqq k}\right)^{n}\right\rangle\right\rangle-\#[\text { pairings of }\{1, \ldots, n\}] \cdot\left\langle\left\langle F_{\leqq k}^{2}\right\rangle\right\rangle^{n / 2},
$$

vanish in the limit $N \rightarrow \infty$ :

$$
R_{k, n}(N) \underset{N \rightarrow \infty}{\longrightarrow} 0 \text { for all } k \text { and } n>0 .
$$

First, we note that as square-integrable functions of $\left\{J_{i j}\right\}$ the weights $w(\gamma)$ are orthogonal for different loops [with respect to the measure $P(d J)$ ]. By easy counting

$$
\begin{gathered}
\sum_{\gamma:|\gamma|=k}\left\langle\left\langle w(\gamma)^{2}\right\rangle\right\rangle=\frac{N(N-1)(N-2) \ldots(N-k+1)}{2 k}\left(\left\langle\left\langle\left(\tanh \frac{\beta J_{12}}{\sqrt{N}}\right)^{2}\right\rangle\right\rangle\right)^{k} \\
\substack{\nearrow \rightarrow \infty) \\
(N \rightarrow \infty} \\
\frac{\left(\beta^{2} J^{2}\right)^{k}}{2 k},
\end{gathered}
$$

which directly implies (3.8) and (3.9). What is left is the main part of the analysis: dealing with the higher moments. These, however, are considerably simplified by the introduction of the finite cutoff $k$.

To prove iii) let us write

$$
\begin{aligned}
R_{k, n}(N)= & \left.\sum_{\substack{\gamma_{1}, \ldots, \gamma_{n} \\
\left|\gamma_{2}\right| \leqq k}}\left\langle w\left(\gamma_{1}\right) \ldots w\left(\gamma_{n}\right)\right\rangle\right\rangle \\
& \left.-\sum_{\substack{\text { pairings } \\
\text { of }\{1, \ldots, n\}}} \sum_{\substack{\gamma_{1}, \ldots, \gamma_{n} \\
\left|\gamma_{i}\right| \leqq k}}\left\langle\left\langle w\left(\gamma_{i_{1}}\right) w\left(\gamma_{j_{1}}\right)\right\rangle\right\rangle \ldots\langle w w\rangle\right\rangle,
\end{aligned}
$$

and group the terms in the above expression according to $\Gamma=\gamma_{1} \circ \ldots \circ \gamma_{n}$, by which we denote the multi-graph whose multiplicity for each bond is the number of loops $\gamma_{i}$ containing that bond.

For $\Gamma$ which consist of non-intersecting "double loops" the structure of the contributing $n$-tuples $\gamma_{1}, \ldots, \gamma_{n}$ is unambiguous - up to permutations. To each such permutation there corresponds exactly one term in each of the sums over $\left\{\gamma_{1}, \ldots, \gamma_{n}\right\}$ in (3.13), and there is exactly one pairing in the second sum with nonvanishing $\langle\langle w \cdot w\rangle\rangle \ldots\langle\langle w \cdot w\rangle\rangle$, which then equals $\langle\langle w(\Gamma)\rangle\rangle$. The two terms clearly cancel each other, and hence the net contribution of the double loop graphs to $R$ is zero.

By an easy estimate of the remaining contributions, one gets

$$
\left|R_{k, n}(N)\right| \leqq \sum_{\Gamma}^{*} c(\Gamma) \omega(\Gamma),
$$

with

$$
\omega(\Gamma)=\prod_{i<j}\left\langle\left\langle\left(\frac{\beta^{2} J_{12}^{2}}{N}\right)^{n_{t_{J} / 2}}\right\rangle\right\rangle \leqq\left[\beta\left\langle\left|J_{12}\right|^{n}\right\rangle^{1 / n}\right]^{|\Gamma|} \cdot N^{-|\Gamma| / 2} .
$$

The summation $\left(^{*}\right)$ is over multi-graphs, each of which is given by the set of multiplicities $\left\{n_{i j} \mid 1 \leqq i<j \leqq N\right\}$, such that:

1) $|\Gamma| \equiv \sum_{i<j} n_{i j}(\Gamma) \leqq n \cdot k$, 
2) for each bond with $n_{i j}(\Gamma) \neq 0$, and each vertex with nonvanishing order $\hat{n}_{i}(\Gamma)$ $\equiv \sum_{j(\neq i)} n_{i j}(\Gamma)$,

$$
2 \leqq n_{i j}(\Gamma) \leqq n, \quad \text { and } \quad \hat{n}_{i}(\Gamma) \geqq 4,
$$

3) there is either a vertex with $\hat{n}_{i}(\Gamma) \geqq 8$, or a pair of vertices each having $\hat{n}_{i}(\Gamma) \geqq 6$.

(The last two properties follow from the fact that $\Gamma$ is a union of simple loops, has no single bonds, and is not decomposable into a collection of nonintersecting double loops.)

The coefficient $c(\Gamma)$ in (3.14) is a combinatorial factor satisfying

uniformly in $N$.

$$
c(\Gamma) \leqq a(k, n),
$$

For each $n$ and $k$, there is only a uniformly finite number of equivalence classes of graphs $\Gamma$, modulo permutations of $\{1, \ldots, N\}$, which contribute to the sum in (3.14). For each such class the weight associated with each graph decays with $N$ as const $\cdot N^{-|\Gamma| / 2}$, while the number of elements in the class grows proportionally to $N^{V(\Gamma)}$, where $V(\Gamma)$ is the number of vertices of $\Gamma$. However the above restrictions 2 ) and 3) on $\Gamma$ imply that

$$
|\Gamma|=\frac{1}{2} \sum_{i} \hat{n}_{i}(\Gamma) \geqq 2 V(\Gamma)+2,
$$

i.e.

$$
|\Gamma| / 2-V(\Gamma) \geqq 1
$$

Therefore we obtain

$$
\left|R_{k, n}(N)\right| \leqq \tilde{a}(k, n) / N,
$$

which, by the criterion $\mathrm{C} 1$ of Sect. 2 - and by the known structure of the Gaussian moments, implies that $F_{\leqq k}$ converges in distribution to a Gaussian, whose variance is easily computed [as in (3.12)] to be

$$
\left\langle v_{k}^{2}\right\rangle=\sum_{m=3}^{k} \frac{\left(\beta^{2} J^{2}\right)^{m}}{2 m} .
$$

The claim made in (3.3) follows now by the convergence criterion C3.

The other two statements, (3.4) and (3.5), are much more elementary. Equation (3.4) is equivalent (in its $L^{2}$ sense) to:

$$
\mathrm{A} v\left(\left(\sum_{\gamma} w(\gamma)^{2}-\left\langle v^{2}\right\rangle\right)^{2}\right) \underset{N \rightarrow \infty}{\longrightarrow} 0,
$$

which is easy to prove by the arguments used above. For (3.5) we take any $k>\min \{2,3 / \varepsilon\}$ and use the bound,

$$
\begin{aligned}
\operatorname{Prob}\left(\max _{\gamma}\{|w(\gamma)|\} \geqq \frac{1}{N^{3 / 2-\varepsilon}}\right) & \leqq \sum_{\gamma}\left\langle\left\langle|w(\gamma)|^{k}\right\rangle \cdot N^{(3 / 2-\varepsilon) \cdot k}\right. \\
& \leqq \frac{x^{3} / 6}{1-x \cdot N^{1-\frac{k}{2}}} \cdot N^{-(\varepsilon k-3)} \underset{N \rightarrow \infty}{\longrightarrow} 0,(3.18)
\end{aligned}
$$


where $x=\beta^{k}\left\langle\left\langle\left|J_{12}\right|^{k}\right\rangle\right\rangle$, and the summation is performed just as in (3.12) (with the dominant term corresponding to $|\gamma|=3$ ).

Before we turn to the partition function, let us note the following consequence of the above limits.

Lemma 3.2. For $\beta J<1$,

$$
\prod_{\gamma}[1+w(\gamma)] \underset{N \rightarrow \infty}{\stackrel{\mathscr{D}}{\longrightarrow}} \exp \left(v-\frac{1}{2}\left\langle v^{2}\right\rangle\right)
$$

where $v$ is the same Gaussian variable as in (3.3) and (2.14).

(To prove it, one may start by rewriting each factor, using: $1+x$ $=\exp \left[x-\frac{1}{2} x^{2}+O\left(x^{3}\right)\right]$, for $x<\frac{1}{2}$, and then substituting (3.3)-(3.6) of Lemma 3.1.)

Let us now turn to the reduced partition function $\hat{Z}$. Our first step is to reduce the collection of graphs with which one need be concerned. Because of its general usefulness, the following estimate is stated separately.

Lemma 3.3. For $\beta J<1$, the contribution to $\hat{Z}$ of large graphs decays exponentially, in the following sense,

$$
\left\langle\left\langle\left(\sum_{\substack{\text { simple closed } \\|\Gamma| \geqq k}} w(\Gamma)\right)^{2}\right\rangle\right\rangle \leqq \text { const } e^{\sqrt{2 k}} \cdot\left(\beta^{2} J^{2}\right)^{k} .
$$

Proof. By the orthogonality of the different graphs' weights,

$$
\text { L.H.S. }=\sum_{\substack{\Gamma \text { simple closed } \\|\Gamma| \geqq k}}\left\langle\left\langle w(\Gamma)^{2}\right\rangle\right\rangle \text {. }
$$

For given sufficiently large $k$, let $\varepsilon=\varepsilon(k)>0$ be defined by $e^{\varepsilon}(\beta J)^{2}=1-1 / \sqrt{2 k}$. We have

$$
\begin{aligned}
& \sum_{\substack{\Gamma \text { simple closed } \\
|\Gamma| \geqq k}}\left\langle\left\langle w(\Gamma)^{2}\right\rangle\right\rangle e^{-\varepsilon k} \prod_{\text {loops } \gamma}\left(1+\left\langle\left\langle w(\gamma)^{2}\right\rangle\right\rangle e^{\varepsilon|\gamma|}\right) \\
& \leqq e^{-\varepsilon k} \exp \left[\sum_{m=3}^{\infty} \frac{1}{2 m}\left(e^{\varepsilon} \beta^{2} J^{2}\right)^{m}\right] \\
& \leqq\left(\beta^{2} J^{2}\right)^{k} \exp \left[-k \ln \left(e^{\varepsilon} \beta^{2} J^{2}\right)+\frac{1}{2} \frac{1}{1-e^{\varepsilon} \beta^{2} J^{2}}\right] \\
& \leqq \text { const } e^{\sqrt{2 k}}\left(\beta^{2} J^{2}\right)^{k} .
\end{aligned}
$$

Putting together the various estimates obtained above, we shall now prove the main result of this subsection.

Proof of Proposition 2.2. i) Let

$$
\varphi(z)=\prod_{\gamma, \text { simple loops }}[1+z w(\gamma)] .
$$

This function - which depends on $N$ and $\{\beta J\}$, is a polynomial in $z$. Using Lemma 3.1, and the remainder formula,

$$
\left|\varphi(1)-\sum_{n=0}^{m} \frac{1}{n !} \varphi^{(n)}(0)\right| \leqq \frac{R}{R-1} \frac{1}{R^{m+1}} \sup _{\substack{z \in \mathbb{C} \\|Z|=R}}|\varphi(z)|
$$


(for a function which is analytic in a disk of radius $R>1$ ) which we apply with a fixed $R>1$, it is easy to see that

$$
\prod_{\gamma}[1+w(\gamma)]-\sum_{n=0}^{m_{N}} \sum_{\substack{\left\{\gamma_{1}, \ldots, \gamma_{n}\right\} \\ \gamma_{t} \neq \gamma_{j} ;\left|\gamma_{l}\right| \leqq k_{N}}} w\left(\gamma_{1}\right) \cdot \ldots \cdot w\left(\gamma_{n}\right) \underset{N \rightarrow \infty}{\stackrel{D}{\longrightarrow}} 0
$$

(where the sum is over $n$-tuples of distinct loops), provided $k_{N}$ and $m_{N}$ diverge when $N \rightarrow \infty$.

At the same time, by the arguments which were used in the proof of Lemma 3.1,

$$
\begin{aligned}
\| \hat{Z} & -\sum_{n=0}^{m} \sum_{\substack{\left\{\gamma_{1}, \ldots, \gamma_{n}\right\} \\
\gamma_{t} \neq \gamma_{j} ;\left|\gamma_{2}\right| \leqq k}} w\left(\gamma_{1}\right) \cdot \ldots \cdot w\left(\gamma_{n}\right) \|_{2}^{2} \\
& \leqq \hat{C}(m \cdot k) / N+\sum_{\substack{\Gamma \text { simple closed } \\
|\Gamma| \geqq m \cdot k}}\left\langle\left\langle w(\Gamma)^{2}\right\rangle\right\rangle \\
& \leqq \hat{C}(m \cdot k) / N+C^{\prime} \exp \left[-\ln (\beta J)^{-2} \cdot m k+\sqrt{m k}\right],
\end{aligned}
$$

where $\hat{C}$ is a finite quantity which is independent of $N$, and the last step is by Lemma 3.3.

With $m$ and $k$ chosen so that $m, k \rightarrow \infty$ while $\hat{C}(m \cdot k) / N \rightarrow 0$ (as $N \rightarrow \infty$ ), the estimates (3.25) and (3.26) show that

$$
\hat{Z}_{N}-\prod_{\gamma}[1+w(\gamma)] \stackrel{\mathscr{D}}{\longrightarrow} 0
$$

which together with Lemma 3.2 imply that the distribution of $\hat{Z}_{N}$ tends to that of the log-normal variable described by (2.13).

ii) To complete the derivation of the fluctuations in the total free energy, we note that by the decomposition (2.12),

$$
\begin{aligned}
Z /\left(2^{N} \hat{Z}\right) & =\exp \left(\sum_{i<j} \log \cosh \left(\beta J_{i j} / \sqrt{N}\right)\right) \\
& =\exp \left(\sum_{i<j}\left[\frac{1}{2 N} \beta^{2} J_{i j}^{2}-\frac{2}{4 ! N^{2}} \beta^{4} J_{i j}^{4}\right]+O(1 / N)\right) \\
& =\exp \left((N-1) \frac{1}{4} \beta^{2} J^{2}-\frac{1}{4 !} \beta^{4}\left\langle\left\langle J_{12}^{4}\right\rangle\right\rangle+\frac{1}{2 N} \sum_{i<j} \beta^{2}\left(J_{i j}^{2}-J^{2}\right)+O\left(\frac{1}{N}\right)\right) \\
& =\left\langle\left\langle Z / 2^{N}\right\rangle\right\rangle \cdot \exp \left(\tilde{u}-\frac{1}{2}\left\langle\left\langle\tilde{u}^{2}\right\rangle\right\rangle+O\left(\frac{1}{N}\right)\right),
\end{aligned}
$$

where in the last step we use (2.4)-(2.5), and $\tilde{u}$ is the random variable

$$
\tilde{u}=\sum_{i<j} \beta^{2}\left(J_{i j}^{2}-J^{2}\right) /(2 N) .
$$

By the central limit theorem, $\tilde{u}$ converges in distribution to a Gaussian variable [with variance $\left.1 / 8 \cdot \beta^{4}\left(\left\langle J_{12}^{4}\right\rangle\right\rangle-J^{4}\right)$ ]. Note that for each $N$ the variable $\tilde{u}$ is orthogonal, in the $\left\langle\langle\cdot\rangle\right.$ sense, to the quantity $\sum_{\gamma} w(\gamma)$ - which is the source of the residual fluctuations in $\log \hat{Z}$. Using the analysis of Lemma 3.1 it is easy to show 
that in the limit $N \rightarrow \infty$ the two fluctuation terms in $\log Z$ add as a pair of independent Gaussians (as suggested by their orthogonality). The result is that the distribution of $\log Z$ tends to the $\log$-normal law described in Proposition 2.2.

\section{Mean Free Energy and an Order Parameter}

The preceding discussion yielded an explicit form for the distribution of the total free energy. In this section we step back, in a sense, and use just the leading order of that law for a proof of the result of SK and TAP on the free energy per site, for $\beta J<1$ (a conditition identified by TAP).

Let us start with a useful thermodynamic relation, which implies that any deviation in the free energy density of the "quenched state" from that of the "annealed system" [i.e. any violation of (2.6)] is necessarily associated with the nonvanishing of the order parameter defined by (2.7).

Proposition 4.1. For each $N$ and $\beta$,

$$
\frac{d}{d \beta} \frac{1}{N}\left\langle\left\langle\log Z_{N}(\{\beta J\})\right\rangle\right\rangle=\frac{1}{2} \beta J^{2}\left(1-\left\langle\left\langle\tau_{N}(\{\beta J\})\right\rangle\right\rangle\right)+R_{N},
$$

where $R_{N}$ is a remainder term which vanishes as $N \rightarrow \infty$, satisfying

$$
R_{N} \leqq \operatorname{const} \beta^{2} / \sqrt{N} .
$$

Proof. Standard differentiation gives

$$
\frac{d}{d \beta} \frac{1}{N} \log Z_{N}(\{\beta J\})=\frac{1}{N} \sum_{i<j} \frac{J_{i j}}{\sqrt{N}}\left\langle\sigma_{i} \sigma_{j}\right\rangle_{\{\beta J\}},
$$

where, as we shall see, the weak correlation of $\left\langle\sigma_{i} \sigma_{j}\right\rangle$ with the direct coupling $J_{i j}$ is significant. Denoting (with some abuse of notation) by $\left\langle\sigma_{i} \sigma_{j}\right\rangle_{0}$ the expected value in a system for which the coupling $J_{i j}$ is set equal to zero, we have

$$
\begin{aligned}
\left\langle\sigma_{i} \sigma_{j}\right\rangle & =\frac{\tanh \left(\beta J_{i j} / \sqrt{N}\right)+\left\langle\sigma_{i} \sigma_{j}\right\rangle_{0}}{1+\left\langle\sigma_{i} \sigma_{j}\right\rangle_{0} \tanh \left(\beta J_{i j} / \sqrt{N}\right)} \\
& =\left\langle\sigma_{i} \sigma_{j}\right\rangle_{0}+\frac{\beta J_{i j}}{\sqrt{N}}\left(1-\left\langle\sigma_{i} \sigma_{j}\right\rangle_{0}^{2}\right)+O\left(\frac{\beta^{2} J_{i j}^{2}}{N}\right) .
\end{aligned}
$$

Hence,

$$
\begin{gathered}
\frac{1}{N} \frac{d}{d \beta} \log Z_{N}(\{\beta J\})-\frac{1}{N^{2}} \sum_{i<j} \beta J_{i j}^{2}\left(1-\left\langle\sigma_{i} \sigma_{j}\right\rangle_{0}^{2}\right) \\
\quad=\frac{1}{N^{3 / 2}} \sum_{i<j} J_{i j}\left\langle\sigma_{i} \sigma_{j}\right\rangle_{0}+\widetilde{R}_{N}
\end{gathered}
$$

with a reminder $\widetilde{R}_{N}$ which satisfies a bound like in (4.2). Since the average (over $J$ ) of the first term on the right side of (4.4) vanishes, the above expressions directly yield (4.1). 
Proof of Proposition 2.1. Let

$$
Q(\beta):=\lim _{N \rightarrow \infty} \frac{1}{N} \log \left\langle\left\langle Z_{N}\right\rangle\right\rangle=\log 2+\frac{1}{4} \beta^{2} J^{2} .
$$

We shall now obtain the three claims presented in Proposition 2.1 - in reverse order.

iii) Taking just the leading order of the result of Proposition 2.2 (ii) on the total free energy, we obtain:

$$
\frac{1}{N} \log Z_{N}(\{\beta J\})-Q(\beta) \underset{N \rightarrow \infty}{\stackrel{\mathscr{D}}{\longrightarrow}} 0, \text { for all } \quad \beta J>1
$$

which is an equivalent form of (2.10). (The $L^{p}$ convergence is proven along with ii).)

ii) In order to deduce from (4.6) the convergence of the mean values (i.e. $\langle\langle\ldots\rangle\rangle)$, one has to determine that they are not affected by some large fluctuations whose probability vanishes in the limit. The following two general bounds show that the distribution of the free energy per site is sufficiently compact; for example, it certainly satisfies the criterion C2 presented in Sect. 2.

1) For all $\{\beta J\}$

$$
\frac{1}{N} \log Z_{N}(\{\beta J\}) \geqq \log 2,
$$

which follows from:

$$
Z_{N}(\{\beta J\}) / 2^{N}=\operatorname{tr} e^{-\beta H_{J}(\sigma)} \geqq \exp \left(-\operatorname{tr} \beta H_{J}(\sigma)\right)=1 .
$$

And, in the other direction,

2) for all $\beta$

$$
\operatorname{Prob}_{J}\left(\frac{1}{N} \log Z_{N}(\{\beta J\}) \geqq Q_{N}(\beta)+\varepsilon\right) \leqq e^{-\varepsilon N},
$$

where $Q_{N}(\beta)$ is the finite volume approximant of $Q(\beta)$. (The last bound is derived by considering the mean value of $Z$.)

As explained above, the two bounds (4.8) and (4.9) imply that, at any temperature, $\mathscr{D}$-convergence of the free energy per site implies also the convergence of the mean values. Hence, we may deduce from (4.6) the relation (2.9), as well as the following stronger statement,

$$
\left\langle\left\langle\left|\frac{1}{N} \log Z_{N}-Q(\beta)\right|^{p}\right\rangle\right\rangle \underset{N \rightarrow \infty}{\longrightarrow} 0, \text { for all } p<\infty .
$$

i) By Proposition 4.1, the derivative with respect to $\beta$ of the left side of $(4.10)$ is nonpositive for all $\beta \geqq 0$. The vanishing of the limit for $\beta<1 \mathrm{implies,} \mathrm{therefore,} \mathrm{that}$ also the derivative tends to zero, as $N \rightarrow \infty$. By the explicit formula (4.1), the last statement means that

$$
\lim _{N \rightarrow \infty}\left\langle\left\langle\tau_{N}(\{\beta J\})\right\rangle\right\rangle=0, \text { for all } \beta J<1,
$$

proving the first assertion in Proposition 2.1. 
It might be interesting to note that the above proof, together with the monotonicity mentioned below, implies that for each open set in the range of $\beta$ the three conditions listed in Proposition 2.1 are in fact equivalent.

Formula (4.1) carries also the following implications for the order parameter $\tau$ in the low temperature regime.

Proposition 4.2. i) For $\beta J>1$

$$
\mathscr{D}-\limsup _{N \rightarrow \infty} \tau_{N}(\{\beta J\}) \leqq 1-\frac{1}{\beta J},
$$

and in general $\mathscr{D}$-lim $\sup _{N \rightarrow \infty} \beta\left[1-\tau_{N}(\{\beta J\})\right]$ (as well as other well defined $\mathscr{D}$-limits) are increasing functions of $\beta$.

ii) $\left\langle\left\langle\tau_{N}(\{\beta J\})\right\rangle\right\rangle$ obeys the lower bound (2.21).

$$
\mathscr{D}-\liminf _{N \rightarrow \infty} \tau_{N}(\{\beta J\})=1-O(1 / \beta), \quad \text { as } \quad \beta \rightarrow \infty .
$$

Proof. i) By general principles the left side of (4.1) is a monotone increasing function of $\beta$. Equation (4.12) is a consequence of this monotonicity and the fact that this function vanishes for all $\beta J<1$.

ii), iii) Since the left side of (4.1) equals the negative of the mean value of the energy per site, $e_{J}(\beta)=\left\langle H_{J}(\sigma) / N\right\rangle_{\beta J}$ (which is typically negative), (4.1) may be rewritten as follows

$$
\left\langle\left\langle\tau_{N}(\{\beta J\})\right\rangle=1+\frac{2\left\langle\left\langle e_{J}(\beta)\right\rangle\right\rangle}{\beta J^{2}}-O(\beta / \sqrt{N}) .\right.
$$

This directly implies the lower bound (2.21). Equation (4.13) is obtained by combining (4.14) with any bound on $\left|\left\langle e_{J}\right\rangle\right\rangle \mid$, e.g. an $L^{1}$ version of (2.20).

\section{Some Weak Bounds for the Free Energy at Low Temperatures}

The thermodynamic arguments mentioned in the introduction show that the situation described by Proposition 2.1 does not persist at low temperatures. The phase transition is generally expected to occur at $\beta J=1[3,6]$. [Note that this is the point at which the $O(1)$ fluctuations of the total free energy, in Proposition 2.2, diverge.] The transition reflects the fact that for low (negative) values of $e$, the number of spin configurations with energy densities $H_{J}(\sigma) / N \approx e$ is lower than what is obtained from an extrapolation of the high temperature entropy, which is $S(e) / N$ $=\log 2-(e / J)^{2}$ [computed by a Legendre transform of $\left.P(\beta)\right]$. In this section we present some explicit bounds whose purpose is to show that $S(e)$ does not vanish too fast. While the results are not very strong, and they do not deal with the structure discussed in $[5,6,8,9]$, they demonstrate two algorithms for the construction of some low energy configurations.

It might be remarked first that for any fixed configuration $\sigma$ the energy per site, $H_{J}(\sigma) / N$, is vanishingly small for "typical" $J$, since

$$
\left\langle\left\langle H_{J}(\sigma)^{2}\right\rangle\right\rangle^{1 / 2}=\left(\sum_{i<j} J^{2} / N\right)^{1 / 2}=O(\sqrt{N}) .
$$

Hence the low energy configurations do have a nontrivial $J$ dependence. 


\section{a) A Sequential Algorithm}

For a fixed set of couplings $\left\{J_{i j}\right\}$, let us associate to each spin configuration $\sigma$ $\left(=\left\{\sigma_{i}\right\}\right)$ a sequence of integers $\eta_{i}= \pm 1$, defined by

$$
\begin{gathered}
\eta_{1}=\sigma_{1}, \quad \text { and for } j>1, \\
\eta_{j}= \begin{cases}\sigma_{j} \operatorname{sgn} \sum_{i=1}^{j-1} J_{i j} \sigma_{i} & \text { if } \sum_{i=1}^{j-1} J_{i j} \sigma_{i} \neq 0 \\
\sigma_{1} \sigma_{j} & \text { otherwise. }\end{cases}
\end{gathered}
$$

The energy can now be expressed as

$$
H_{J}(\sigma)=-\sum_{k=1}^{N} A_{k}(\sigma, J) \eta_{k}(\sigma, J)
$$

where $A_{k}$ is the nonnegative quantity

$$
A_{k}=\left|\sum_{i=1}^{k-1} \frac{J_{i j}}{\sqrt{N}} \sigma_{i}\right| .
$$

The distribution of $A_{k}$ at any fixed $\sigma$ is, for $k$ of the order of $N$, asymptotically given by the density

$$
P_{k}(A)= \begin{cases}\frac{2}{\sqrt{2 \pi \frac{k-1}{N} J^{2}}} \exp \left(-\frac{N}{k-1} \frac{A^{2}}{2 J^{2}}\right) & \text { for } A \geqq 0 \\ 0 & \text { for } A<0\end{cases}
$$

(by the central limit theorem), with the mean value

$$
\begin{aligned}
\alpha_{k}^{(N)}: & =\left\langle\left\langle A_{k}(\sigma, J)\right\rangle \quad(\text { at a fixed } \sigma)\right. \\
& \approx\left(\frac{2}{\pi} \frac{k-1}{N}\right)^{1 / 2} J .
\end{aligned}
$$

For a fixed set of couplings $J$, the map $\sigma \rightarrow \eta$ is invertible (by a simple procedure in which $\sigma_{i}$ are recovered in the order $\left.i=1,2, \ldots\right)$, and its inverse defines for us $\sigma(\eta, J)$. We now claim that for a large collection of $\eta$, e.g. $\eta \equiv+1$, the energy at fixed $\eta$ is for "typical" $J$ not $O(\sqrt{N})$, as it is at fixed $\sigma$ [by (5.1)], but is instead of order $O(N)$ - with an $O(\sqrt{N})$ fluctuation with respect to the quantity

$$
E(\eta)=-\sum_{k=1}^{N} \alpha_{k}^{(N)} \eta_{k}
$$

Lemma 5.1. For each fixed $\eta$

$$
\left\langle\left\langle\left(H_{J}(\sigma(\eta, J))-E(\eta)\right)^{2}\right\rangle \leqq J^{2} \frac{N-1}{2} .\right.
$$

Proof. For a fixed $\eta$, the quantities $\sigma_{1}, \ldots, \sigma_{k-1}$ and $A_{1}, \ldots, A_{k-1}-$ as functions of $(\eta, J)$ depend on only those couplings $J_{i j}$ for which $i, j<k$. Yet when all those 
couplings (i.e. not just $a_{2}, \ldots, a_{k-1}$ ) are specified, the distribution of

$$
\sum_{i=1}^{k-1} \frac{J_{i k}}{\sqrt{N}} \sigma_{i}
$$

is independent of their values, since those affect this quantity only through the variables $\sigma_{i}$, and the distribution of $J_{\cdot, k}$ is independent of the previous $J$ 's and is invariant under reflection "gauge transformations." It follows that the random variables $A_{k}=A_{k}(\sigma(\eta, J), J)$ are jointly independent (!), and their distributions do not even depend on $\eta$.

By the above independence property, the left side of (5.8) is

$$
\begin{aligned}
\left\langle\left\langle\left[\sum_{k=1}^{N}\left(A_{k}-\left\langle\left\langle A_{k}\right\rangle\right)\right) \eta_{k}\right]^{2}\right\rangle\right\rangle & =\sum_{k=1}^{N}\left\langle\left\langle\left(A_{k}-\left\langle\left\langle A_{k}\right\rangle\right\rangle\right)^{2}\right\rangle\right\rangle \\
& \leqq \sum_{k=1}^{N} \sum_{j=1}^{k-1} \frac{J^{2}}{N}=J^{2}(N-1) / 2,
\end{aligned}
$$

where the actual behavior of the left side is $\approx(1-2 / \pi) J^{2}(N-1) / 2$ [using (5.6)].

As $\eta$ is varied, $E_{N}(\eta) / N$ varies over the interval $\left[-e_{N}^{*}, e_{N}^{*}\right]$, where $[$ by $(5.6)]$

$$
\lim _{N \rightarrow \infty} e_{N}^{*}=\int_{0}^{1}\left(\frac{2}{\pi} \alpha\right)^{1 / 2} J d \alpha=\sqrt{\frac{2}{\pi}} \frac{2}{3} J \cong 0.53 J .
$$

Hence the lower bound (2.23) on $e_{0}(\{J\})$.

The above procedure may be used to generate a large number of low energy configurations. The corresponding lower bound for the free energy at finite $\beta$ is stated in Proposition 2.3, which we are now ready to derive.

Proof of Proposition 2.3. Let us recall the standard variational principle, according to which (for each $J$ )

$$
\log Z_{N}(\{\beta J\})=\max \left\{\sum_{\sigma} \varrho(\sigma) \log \varrho(\sigma)^{-1}-\beta \sum_{\sigma} \varrho(\sigma) H_{J}(\sigma) \mid \varrho \geqq 0, \sum_{\sigma} \varrho(\sigma)=1\right\} .
$$

For a given $J$ we choose to consider the variational state

$$
\tilde{\varrho}_{\{\beta J\}}(\sigma)=\frac{e^{-\beta E(\eta(\sigma, J))}}{Z_{N}^{*}(\beta)},
$$

with the $\{J\}$-independent normalizing factor

$$
\begin{aligned}
Z_{N}^{*}(\beta) & =\sum_{\sigma} e^{-\beta E(\eta(\sigma, J))}=\sum_{\eta} e^{-\beta E(\eta)} \\
& =2^{N} \prod_{k=1}^{N} \cosh \left(\beta \alpha_{k}^{(N)}\right) .
\end{aligned}
$$

The substitution of $\tilde{\varrho}_{\{\beta J\}}(\cdot)$ in (5.11) yields the bound

$$
\frac{1}{N} \log Z_{N}(J) \geqq \frac{1}{N} \log Z_{N}^{*}(\beta)-Q_{N}(\{\beta J\}),
$$


with

$$
Q_{N}(\{\beta J\})=\frac{\beta}{N} \sum_{\eta} \frac{e^{-\beta E_{N}(\eta)}}{Z_{N}^{*}(\beta)}\left[H_{J}(\sigma(\eta, J))-E_{N}(\eta)\right] .
$$

By Lemma 5.1, and the triangle inequality,

$$
\left\langle\left\langle Q_{N}(\{\beta J\})^{2}\right\rangle\right)^{1 / 2} \leqq \beta J / \sqrt{2 N},
$$

and therefore $\mathscr{D}-\lim _{N \rightarrow \infty} Q_{N}(\{\beta J\})=0$. Using (5.6) and (5.13) to evaluate $N^{-1} \log Z_{N}^{*}$, we see that (5.14) reduces as $N \rightarrow \infty$ to the claimed inequality (2.22).

\section{b) Shadowing the Ground State of $J_{i j}$}

Another algorithm (less explicit than the above) for generating low energy spin configurations is to choose $\sigma$ which maximizes the overlap with one of the low eigenstates of the symmetric matrix $M_{i, j}=J_{i j} /(2 \sqrt{N})$ - for which we generate diagonal terms with the same distribution as the other $J$ 's. The Hamiltonian (1.1) may be expressed by means of $M$ (with $M_{i, i}=0$ ) as

$$
H_{J}(\sigma)=-\sum_{i, j} \sigma_{i} M_{i, j} \sigma_{j}+\sum_{i} J_{i i} /(2 \sqrt{N})=\sum_{\alpha} \lambda_{\alpha}\left\langle\varphi^{\alpha} \mid \sigma\right\rangle^{2}+O(1)
$$

where $\varphi^{\alpha}$ are normalized eigenvectors of $M$ and $\lambda_{\alpha}$ are the corresponding eigenvalues.

By Wigner's semicircle law, which has been extended also to other than Gaussian ensembles [10], the eigenvalues $\lambda_{\alpha}$ are asymptotically distributed with density

$$
C \sqrt{J^{2}-u^{2}} d u
$$

over the interval $[-J, J]$. Let $\tilde{\varphi}$ correspond to the lowest eigenvalue, or any other "fixed" eigenvalue with $\tilde{\lambda} \approx-J$. The components $\tilde{\varphi}_{i}$ of $\tilde{\varphi}$ have, approximately, a Gaussian distribution with mean 0 and variance $1 / N$. Choosing $\sigma_{i}=\operatorname{sgn} \tilde{\varphi}_{i}$, we have

$$
\langle\tilde{\varphi} \mid \sigma\rangle \approx N \int_{-\infty}^{\infty}|x| e^{-N x^{2} / 2} \sqrt{\frac{N}{2 \pi}} d x=2 \sqrt{\frac{N}{2 \pi}},
$$

and hence

$$
\langle\tilde{\varphi} \mid \sigma\rangle^{2} \approx \frac{2}{\pi} N \cong 0.64 N
$$

We also argue that if $\sigma$ is chosen by the above criterion then for typical $J$ 's the contribution to $H_{J}(\sigma)$ from all the other eigenvectors in the expansion (5.17) is of order $O(\sqrt{N})$. In the Gaussian case that is easily seen from the invariance of the distribution of the frame of all the eigenvectors under general unitary rotations even when it is conditioned on the spectrum. By this invariance, a bound like (5.1) applies also to $H_{J}(\sigma)-\tilde{\lambda}\langle\tilde{\varphi} \mid \sigma\rangle^{2}$, even when $\sigma$ is allowed to depend on $H$ through the eigenvector $\tilde{\varphi}$. 
Therefore $e_{0}(J)\langle-(2 / \pi) J\rangle$ (for a "typical" $J$ ), as stated in (2.24). This result is not improved if instead of one "specific" eigenvalue $\tilde{\lambda}$ one takes a finite number of eigenvalues.

\section{References}

1. Sherrington, D., Kirkpatrick, S.: Solvable model of a spin glass. Phys. Rev. Lett. 35, 1792-1796 (1975)

2. Binder, K., Young, A.P.: Spin glasses: Experimental facts, theoretical concepts, and open questions. Rev. Mod. Phys. 54, 801-976 (1986)

3. Thouless, D.J., Anderson, P.W., Palmer, R.G.: Solution of solvable model of a spin glass. Philos. Mag. 35, 593-601 (1977)

4. Feller, W.: An introduction to probability theory and its applications, Vol. II. New York: J. Wiley 1971

5. Derrida, B.: Random energy model: An exactly solvable model of disordered systems. Phys. Rev. B 24, 2613-2626 (1981)

6. Parisi, G.: The order parameter for spin glasses: A function on the interval 0-1. J. Phys. A 13, 1101-1112 (1980)

7. Palmer, R.G., Pond, C.M.: Internal field distribution in model spin glasses. J. Phys. F9, 1451-1459 (1979)

8. Mezard, M., Parisi, G., Virasoro, M.: The replica solution without replicas. Europhys. Lett. 1, 77-82 (1986)

9. Ruelle, D.: A mathematical reformulation of Derrida's REM and GREM. Commun. Math. Phys. 108, 225-239 (1987)

10. Mehta, M.L.: Random matrices. New York, London: Academic Press 1967

Cohen, J., Kesten, H., Newman, C.: Random matrices and their applications. Contemp. Math., Vol. 50. Providence, RI: Am. Math. Soc. 1984

Communicated by E. Lieb

Received March 27, 1986

Note added in proof. We refer the reader to the very interesting recent work by Fröhlich and Zegarlinski [11] for rigorous results on the Gibbs states of long range spin glasses at high temperatures.

2) At the end of the reference list:

11. Fröhlich, J., Zegarlinski, B.: The high-temperature phase of long range spin glasses.

Commun. Math. Phys. 110, 121-155 (1987) 